Revue d'histoire de l'Amérique française

ZAS REVUE D.HISTOIRE DE L'AMÉRIQUE FRANÇAISE

\title{
Sous les auspices du gouvernement canadien
}

Georges-Émile Giguère

Volume 8, numéro 3, décembre 1954

URI : https://id.erudit.org/iderudit/301664ar

DOI : https://doi.org/10.7202/301664ar

Aller au sommaire du numéro

Éditeur(s)

Institut d'histoire de l'Amérique française

ISSN

0035-2357 (imprimé)

1492-1383 (numérique)

Découvrir la revue

Citer cet article

Giguère, G.-É. (1954). Sous les auspices du gouvernement canadien. Revue d'histoire de l'Amérique française, 8(3), 359-379.

https://doi.org/10.7202/301664ar d'utilisation que vous pouvez consulter en ligne.

https://apropos.erudit.org/fr/usagers/politique-dutilisation/ 


\section{SOUS LES AUSPICES DU GOUVERNEMENT CANADIEN}

Une étude plus ou moins prolongée du XIXème siècle canadien nous permet de constater que le gouvernement a plus d'une fois favorisé de son concours financier les manifestations culturelles ou scientifiques de cette époque. La réédition des Relations des Jésuites de la NouvelleFrance est de ce nombre. Elle peut aussi être considérée comme une des plus considérables réalisations de l'imprimerie de toute cette période.

Ces trois gros volumes sortis, en 1858, des ateliers d'"Augustin Côté, éditeur-imprimeur, près de l'archevêché, Québec", se sont vite répandus à travers le pays et même à l'étranger. C'est en vain que le secrétaire d'Etat, Etienne Parent, faisait effectuer des recherches dans les voûtes des Archives du gouvernement à Montréal et dans celles du Bureau de l'Education à Québec pour en trouver un seul exemplaire en 1869.'

Il est peut-être superflu de donner une description d'instruments de travail aussi familiers du chercheur. Certes, il sera plus nouveau de mesurer au moins approximativement les largesses du pouvoir public et de raconter les menus événements qui ont accompagné cette entreprise.

$\mathrm{Au}$ premier volume, on lit dans un "Avis de l'éditeur", trois mentions différentes du Parlement. La première rappelle "l'incendie qui dévora, en 1854, le Parlement, à Québec, avec la riche bibliothèque des chambres législatives", la deuxième mentionne que "le gouvernement canadien nous avait promis de protéger généreusement cette oeuvre importante" et la dernière remercie personnellement "M. G.-B. Faribault, qui eut la douleur de voir réduire en cendres cette bibliothèque américaine". Voilà une abondante matière pour notre recherche. Elle nous

1. Les Archives publiques du Canada nous ont communiqué la copie de quatre lettres appartenant aux archives de la Secrétairerie d'Etat. Elles montrent qu'en septembre 1869, des ordres ont été donnés et des recherches poursuivies, mais sans résultat. 
permettra de reconstituer l'atmosphère de cette époque et de poser en même temps les principaux éléments de solution d'un problème.

En 1850, s'élevait encore à un carrefour de Québec, formé par la côte de la Montagne et les rues de Buade, du Fort et des Remparts, un immense édifice de pierre que l'on continuait d'appeler le "palais épiscopal". ${ }^{2}$ Le Parlement y logeait ses bureaux et sa présence avait attiré dans le voisinage les hommes influents et les principaux journaux de la cité. Les fondations de ce "palais" sont aujourd'hui recouvertes par le parc Montmorency et sa façade était orientée vers le sud, soit dans la direction du Bureau de Postes et du château Frontenac. Dans les rues avoisinantes avaient pris naissance la Gazette des Neilson, le Mercury, le Canadien, le Courrier de Québec, le Journal de Québec et plusieurs autres journaux qui y avaient encore leurs bureaux. En 1857, le Courrier $d u$ Canada ouvrait ses ateliers à deux portes du Journal de Québec et devenait bientôt son adversaire politique. ${ }^{3}$

L'édifice du Parlement était imposant par sa stature et il ne l'était pas moins par son histoire, puisque sa construction remontait à cent cinquante ans. Le gouvernement canadien y avait établi ses dirigeants depuis près de quatre-vingts ans, quand il en fit l'acquisition en 1831 . C'est que cette vaste construction dépassait les besoins d'un évêché et surtout ses revenus. Loué à l'Etat, le palais devenait une source de revenus indispensables à l'administration d'un diocèse aussi étendu que le Canada. L'évêque de Québec, et depuis 1819, l'archevêque, se contentait de quelques appartements particuliers au Séminaire et n'en demeurait pas moins à proximité de sa cathédrale, de son séminaire et du Parlement. ${ }^{4}$

La bibliothèque parlementaire était confiée aux soins d'un conservateur intelligent et industrieux, M. Georges-Barthélemy Faribault, dont le nom et l'emploi s'étaient identifiés l'un à l'autre bien avant l'incendie de 1854. A plusieurs reprises il avait dû réorganiser cette bibliothèque

2. Le Musée provincial de Québec possède une maquette de cet édifice historique. Une photo en a été publiée dans le Rapport de l'archiviste de la Province pour 1931-32, p. 128.

3. Horace Têtu, Historique des journaux de Québec (Québec, 1889). L'auteur affirme que "plus de deux cents journaux, grands et petits, ont vu le jour à Québec".

4. Henri Têtu, Histoire du palais épiscopal de Québec (Québec, 1896). Le chapitre $\mathrm{V}$ est entièrement consacré au Parlement. 
et il en avait publié des catalogues fameux en $1837^{5}, 1845$ et 1852 . Sa réputation était bien établie et elle s'était répandue avantageusement dans les pays étrangers.

Depuis 1834 il occupait ce poste de bibliothécaire parlementaire avec beaucoup de talent et de savoir-faire, quand, en 1846, il sollicita du président de la Chambre, Augustin-Norbert Morin, la reconnaissance officielle de son titre. Le 14 mai 1846, Morin rédigeait la lettre de recommandation que voici :

Je n'hésite pas à témoigner de l'extrême importance des travaux de G.-B. Faribault, Ecuier, pour recueillir des livres rares et des manuscrits sur l'histoire de l'Amérique et du Canada en particulier, dont la collection telle qu'elle est actuellement, se trouve sous ses soins, à la Bibliothèque de la Chambre. ${ }^{6}$

Après l'incendie de 1849 à Montréal et le retour du siège parlementaire à Québec, Faribault voulut refaire sa bibliothèque complètement détruite. Pour son voyage de 1852, il demanda encore à Morin de lui accorder au nom du Parlement les pouvoirs nécessaires. C'est ainsi qu'"une discrétion pleine et entière à exercer dans le choix des livres qui ont rapport à l'Amérique" lui fut accordée par Morin "auprès des institutions publiques, des gouvernements, des sociétés savantes, des bibliothèques publiques, des municipalités, des hommes distingués d'Angleterre et de France". Ce texte demeuré inédit dans les archives provinciales est un hommage implicite à G.-B. Faribault et une véritable révélation sur les dispositions de A.-N. Morin.'

Cela ne nous dit tout de même pas comment les noms de Faribault et de sa bibliothèque ont pu se rattacher à la réédition des Relations en 1856. Si nous examinons le Catalogue de 1837, nous pouvons relever

5. Catalogue d'ouvrages sur l'bistoire de l'Amérique et en particulier sur celle du Canada, de la Louisiane, de l'Acadie et autres lieux (Québec, 1837). Philéas Gagnon mentionne ce catalogue au numéro 357 de son Essai de bibliographie canadienne (Québec, 1896). Voici ce qu'il écrit du Catalogue de 1845: "Ce catalogue est très important. Il nous fait voir l'état de la Bibliothèque avant l'incendie du Parlement. Il fut préparé par les soins de $M$. Faribault. C'est l'exemplaire même de ce dernier, annoté par lui" ( $\left.\mathrm{n}^{\circ} 423\right)$. Cette affirmation garde la même valeur pour le catalogue de 1852 .

6. BRH, 1936, 56.

7. Ce document est conservé aux Archives de la Province de Québec avec la correspondance de Viger à Faribault que nous aurons souvent l'occasion de citer dans la suite. Seules les lettres ont été déchiffrées et transcrites au dactylo. 
quelques mentions des Relations, mais l'auteur fait remarquer qu'il ne s'agissait alors que de citations d'autres catalogues de bibliothèques étrangères. Les signes conventionnels n'indiquent la présence d'aucune Relation au Parlement.

Si nous nous en rapportons à la correspondance échangée entre Jacques Viger et Faribault vers cette époque, il ne semble pas que le bibliothécaire québécois ait possédé d'exemplaires des Relations avant 1842. Si l'incendie du Parlement à Montréal en 1849 paraît en avoir détruit six à dix exemplaires, ce n'est pas avant 1852 que le projet d'en former une collection devient évident. Il y parvenait dès l'année suivante et c'est un fait à remarquer puisque personne avant Faribault n'avait atteint à ce résultat et que jamais après lui aucun bibliophile n'a pu renouveler ce tour de force. ${ }^{8}$

Lors de son voyage en Europe en 1852, Faribault s'était rendu célèbre auprès des savants et des chercheurs en découvrant à Paris les deux "Lettres envoiées..." qui sont précisément les Relations pour les années 1655 et 1659. Tout le monde avant lui s'était laissé tromper par les titres. ${ }^{9}$ On cherchait sous le mot Relations des livres qui étaient intitulés Lettres envoiées. Sa découverte lui faisait honneur et elle complétait une collection dont il pouvait être justement fier. Avouons que son orgueil était légitime. Son ami, Jacques Viger, lui rendait ses hommages fervents par une étude minutieuse qu'il terminait en novembre 1853 sur la fameuse collection du Parlement. Le texte de Viger existe encore en double copie. C'est une "liste des titres de chacun des volumes de la Collection entière des Relations publiées de 1632 à 1672 par les RR. PP. Jésuites du Canada, connues en librairie sous le nom générique de "Relations de la Nouvelle-France" ou de "Relations des Jésuites". Avec notes

8. Toutes les études bibliographiques sur les Relations nous révèlent des lacunes dans les collections. Voir en particulier Justin Winsor, Narrative and Critical History of America (6 vol., Boston et New York, 1884-1886) 4: 296-316. Mêmıe de nos jours, les collections quoique plus rares mais mieux pourvues, ne couvrent pas toute la série. Celle de James Bell comporte soixante-seize exemplaires et celle de McCoy, liquidée l'an dernier, contenait 206 volumes. Pourtant la série n'y était pas entière.

9. Justin Winsor, Narrative and Critical ..., $4: 308-9$. "The rarest of the series"; aussi Léon Pouliot, Etude sur les Relations des Jésuites de la Nouvelle-France (1632-1672), (Montréal, 1940) : 288, note 41. 
\&c. par J.V.".10 Mais la joie du bibliothécaire "vécut ce que vivent les roses: l'espace d'un matin".

Deux mois plus tard, durant la nuit du 1er au 2 février 1854, entre 3 et 4 heures, un incendie ravageait les édifices du Parlement et détruisait une partie de la bibliothèque. Monseigneur Henri Têtu a écrit dans son Histoire du palais épiscopal de Québec: "Le 25 avril 1849, des émeutiers en fureur avaient livré aux flammes le splendide édifice où siégeaient à Montréal les députés de l'Assemblée législative. Un peu moins de cinq ans après, ce fut le tour du Parlement de Québec. Cette fois, il est vrai, ce ne fut pas le fait d'incendiaires, mais la perte ne fut pas moins considérable." "Une grande partie de la bibliothèque a été sauvée, soulignait l'Abeille, petit journal du Séminaire, malheureusement la portion la plus précieuse et la plus difficile à renouveler, celle qui avait coûté, comme on le sait, tant de recherches et de travaux à M. Faribault, est perdue." "' Et, à tour de rôle, tous les journaux, tant de Montréal que de Québec, décrivaient le désastre. Le 7 février suivant, la Minerve de Montréal publiait une lettre venue de Québec:

On a bien sauvé quelques portions de 17,500 volumes qui s'y trouvaient mais dépareillés et gâtés. Hélas! la partie la plus précieuse et la plus regrettable a été la plus malheureuse : notre riche et unique collection sur l'Histoire de l'Amérique, qui s'élevait à 1800 volumes. Je viens de parcourir des monceaux de livres entassés pêle-mêle dans les divers appartements de l'archevêché. Mais hélas! Je n'y ai trouvé que peu d'ouvrages de cette classe, sans un seul volume des Relations des Jésuites. ${ }^{2}$

L'auteur de cette lettre est assez facile à deviner, quoique le journal ne l'ait pas indiqué. Jacques Viger vient confirmer nos conjectures dans une lettre qu'il écrivait à son ami le 10 février 1854 : "J'ai pu faire part de votre lettre à la Minerve, comme vous aurez vu, si vous la recevez."

Remarquons la gradation de cette lettre. Les 17,500 volumes sont "gâtés et dépareillés"; de ce nombre, "la partie la plus précieuse et la

10. La première copie est conservée à la bibliothèque de l'Université Laval (Québec). Elle est reliée avec des ouvrages imprimés. La propreté de ce texte et l'absence de toute rature nous font croire qu'elle ne serait qu'une transcription de celle que possèdent les archives du collège Sainte-Marie (Montréal). L'une et l'autre sont de la main de Jacques Viger, mais la seconde porte des corrections de la main du P. Félix Martin, ami et collaborateur de Viger.

11. Ces deux textes sont tirés du livre de $\mathrm{Mgr}$ Têtu, à la p. 99.

12. Léon Pouliot, Etude sur les Relations des Jésuites, 31. 
plus regrettable (1800 volumes) a été la plus malheureuse". "Mais hélas! je n'y ai trouvé que peu d'ouvrages de cette classe, sans un seul volume des Relations des Jésuites". Et cette dernière partie est soulignée dans le texte. Que répond à cela l'ardent Jacques Viger dans sa lettre ? "Voilà donc encore une fois les Relations des Jésuites détruites, et, cette fois, la seule série complète qu'il y ait peut-être au monde! C'est vraiment du dernier désespoir: le P. Martin est au désol."'13

Quelques jours plus tard, le Journal de Québec glissait dans ses pages deux "petites annonces classifiées" : 14

Toutes personnes ayant en leur possession des livres appartenant à la Bibliothèque du Parlement sont instamment priés [sic] de les remettre au Bibliothécaire sans délai, dans les Bâtiments du Séminaire, joignant le palais de l'Archevêché de Québec. Bâtiments du Séminaire, 13 février 1854.

Livres de la Bibliothèque de la Chambre. On donnera UN PIASTRE pour chaque volume (petits in-12) des "Relations de la Nouvelle-France", qui sera rapporté au Séminaire de Québec d'ici huit jours. On récompensera en proportion ceux qui apporteront d'autres ouvrages sur l'histoire du Canada. 14 février 1854.

Voilà où en étaient à peu près les faits, quand on décida en 1854 de rééditer les historiques Relations des Jésuites.

Le Catalogue de la Bibliothèque du Parlement pour 1858 nous apprend que "lors de l'incendie du 1er février 1854, on n'a pu sauver des flammes que les huit volumes ci-dessous"..' ${ }^{15}$ Devant ces pertes qui avaient toute chance d'être irréparables, on vit se généraliser chez les bibliophiles une certaine crainte. "Quelques amis de notre histoire, écrit l'éditeur de 1858 dans son Avis, désespéraient de retrouver jamais réunis tous les volumes de Relations des Jésuites qui ont rapport à la Nouvelle-France;

13. APQ La correspondance Viger-Faribault s'étend de 1840 à 1858 . Une excellente analyse en a été faite par M. Guy Frégault: "La recherche historique au temps de Garneau (la correspondance Viger-Faribault)" dans le Centenaire de l'Histoire du Canada par François-Xavier Garneau (Montréal, 1945) : 371-90.

14. Ces annonces parurent les $14,16,18$ février et les 2, 4, 7, 9, 16 et 18 mars 1854. On retiendra que ce journal n'était pas quotidien, mais trihebdomadaire.

15. A la page 1191 de ce Catalogue. Ce sont quatre Relations du P. Paul Le Jeune : $1634,1635,1636,1637$, trois du P. Barthélemy Vimont : 1639-40, 1640-41, 1642, et une du P. F.-J. Le Mercier : $1669-70$. 
d'autres, plus confiants, croyaient qu'un sentiment de patriotisme finirait par engager quelque éditeur à rééditer ce monument historique de nos ancêtres." De quel groupe était l'abbé Ferland ? Nous croyons voir une réponse dans ses "Notes sur les registres de N.-D. de Québec" publiées en avril 1854.

Depuis plusieurs années, des accidents déplorables, se succédant avec rapidité, ont détruit beaucoup de documents très précieux pour l'histoire du Canada. Encore quelques pertes semblables et les sources aujourd'hui ouvertes à l'historien auront complètement disparu. Pour prévenir un tel malheur, il serait important de multiplier les copies des manuscrits historiques... Un moyen plus sûr et plus prompt nous est offert par la presse périodique, qui peut prêter un puissant secours à cette oeuvre de préservation, etc $\ldots .^{16}$

A ce moment, certains "désespéraient" et d'autres "plus confiants" suggéraient une réédition. Voilà pourquoi l'éditeur ajoute: "C"est à la sollicitation d'un grand nombre d'entre eux [les amis de l'histoire] que nous avons entrepris de publier une nouvelle édition d'un ouvrage aussi considérable."

Mais l'incendie du Parlement est de février 1854 et la mise en vente des volumes de la réédition est de décembre 1858. Qu'est-ce qui a pu se passer dans l'intervalle ? La première réaction en faveur des Relations, telle que nous venons de la rapporter, s'est-elle maintenue ou est-elle tombée à la rencontre des premières difficultés ? Elle a pu durer pendant quelques mois puisqu'une motion était adoptée en chambre le 2 octobre 1854. Mais une lettre du 1er juin 1855 nous laisse croire que le feu sacré s'était déjà éteint à cette date. "C'est avec beaucoup de regret, écrivait l'historien John Gilmary Shea à l'abbé Ferland, que je vois qu'on a ajourné l'impression des Relations : c'est une honte : même l'état éloigné du Wisconsin publie son histoire documentaire, et pour premier volume donne tout ce qu'il y a dans les Relations sur le territoire entre le lac Michigan et le lac Supérieur. Le Canada doit se réveiller...".'17

16. Ces "Notes" furent publiées d'abord dans le Journal de Québec les 8, 13, 15, 18 avril et les $6,9,11$, et 13 mai 1854 .

17. J. G. Shea venait de commencer avec l'abbé Ferland une correspondance qui devait durer jusqu'à la mort de ce dernier. La plupart de ses lettres sont conservées aux archives de l'archevêché de Québec (AAQ), Armoire E, rayon 11, Papiers Ferland. La collection Gagnon, à la bibliothèque municipale de Montréal, en compte aussi quelques-unes qui ont été signalées par Philéas Gagnon dans son Essai de bibliographie canadienne. La fréquence de ces lettres était d'environ une par mois. Celle que nous venons de citer est à Québec. 
Parmi une série de faits contemporains que nous avons recueillis, et que nous essaierons de regrouper, nous croyons pouvoir distinguer d'abord que cette entreprise n'intéressait au fond que "les amis de l'histoire", donc un petit groupe qui avait plus de coeur que d'argent. La masse du peuple était indifférente en général et son attitude n'éveillait aucun espoir pour le succès d'une souscription populaire. ${ }^{18}$ "Qu'il nous soit permis de dire, écrit l'éditeur, que nous n'avons pu accomplir notre tâche sans de grandes difficultés..."

Sans argent on ne pouvait aller loin. Une lettre du 28 octobre 1853 nous l'apprend. Le P. Félix Martin y écrit: "On ne trouverait pas un libraire ou une librairie qui voulut en [d'une édition] prendre l'entreprise à son compte. Ils consentent seulement à se charger de la vente comme d'une affaire de commission, mais non pour en courir les risques ...". ${ }^{19}$

Que faire en pareilles circonstances? On doit consulter les hommes d'expérience et ceux qui possèdent quelque renom. Faribault a été précisément mis en vedette par les récents événements. Et puis, n'est-il pas président de la Société littéraire et historique de Québec dont la bibliothèque, les archives et le musée, confiés également à ses soins, étaient logés sous le même toit que le Parlement et ont subi les mêmes dommages. $^{20}$ Cette société a du crédit, de l'expérience et du renom. Elle a d'ailleurs obtenu du gouvernement des subventions appréciables pour l'impression de ses documents historiques. ${ }^{21}$ Elle a déjà envoyé, et peutêtre aux frais du gouvernement, plusieurs représentants en France et aux Etats-Unis pour copier et recueillir des documents. Ses archives étaient réputées et elles ont même reçu la visite des consultants américains. Mais la Société littéraire, affirme allégoriquement Faucher de

18. Il ne faut pas oublier que la ville de Québec venait de subir en quelques années deux incendies très désastreux. Des recherches ont révélé que la population la plus affectée se composait de Canadiens français. On peut en affirmer autant de Montréal.

19. ACSM (Archives du collège Sainte-Marie) : Cahier Litterae ad nostros.

20. Henri Têtu, Histoire du palais épiscopal de Québec, 97-100.

21. Voir l'introduction des publications de la Société pour 1838 et 1840. La Chambre avait voté trois cents livres pour favoriser l'impression de ces documents. M. Aegidius Fauteux a étudié le "Mémoire sur les affaires du Canada" dans un intéressant et savant article des Cabiers des dix 5 (1940): 231-92, "Le S . . de C . . . enfin démasqué". 
Saint-Maurice, "était loin d'entendre dans ses coffres le bruissement des flots du Pactole...".22

Autant vaut alors se tourner vers le gouvernement qui en plus d'une occasion déjà a montré sa bienveillance pour l'édition de documents. Il vient tout juste d'autoriser la réédition des Edits et Ordonnances $d u$ Conseil Souverain parce que le tirage de 1803 est épuisé. ${ }^{23}$ N'a-t-il pas encouragé aussi de ses deniers en 1848 l'édition d'une Histoire du Canada en six volumes par Robert Christie ? En 1848 et en 1852, il a participé aux deux premières éditions de celle de F.-X. Garneau. ${ }^{24}$ Et la Société littéraire a obtenu pour sa part deux substantielles subventions pour ses publications de documents en 1838 et 1840 .

En plus de ses titres de président de la Société littéraire et de bibliothécaire du Parlement, Faribault possède en Chambre quelques amis influents. Un de ceux-là est Augustin-Norbert Morin, chef parlementaire du Bas-Canada depuis 1851 qui vient d'être réélu pour former successivement deux ministères dans le gouvernement de coalition: HinksMorin et McNab-Morin. ${ }^{25}$

Cette amitié entre Faribault et Morin a été mise à profit, trois ans plus tôt, lorsqu'ils ont proposé à la Chambre d'éditer une autre Relation d'un jésuite du XVIIème siècle. Ecrite en italien et imprimée à Macerata en 1653, cette Relation venait d'être traduite en français sous le titre de Relation abrégée du P. Bressani, ${ }^{26}$ et son éditeur, le P. Félix Martin, comptait en faire un fort volume in $-8^{\circ}$ de plus de 300 pages avec Avant-propos, notice biographique de l'auteur, notes explicatives et appendices. C'était une oeuvre typographique considérable en raison des clichés d'imprimerie. John Walker, employé chez l'imprimeur Lovell de Montréal, devait

22. Faucher de Saint-Maurice, "Les pages oubliées de notre histoire", dans la Revue canadienne 6(1869) : 401-12. Voir aussi Laverdière, éd., Les Oeuvres de Champlain, préface, p. iv.

23. Une motion a été adoptée en Chambre le 8 décembre 1853.

24. Le Journal de Québec faisait justement remarquer le 11 décembre 1858 que tous ces bénéficiaires des dons du gouvernement n'avaient fait aucune mention des secours reçus pour leurs ouvrages.

25. A. Béchard, L'bonorable A.-N. Morin, (Québec, 1885).

26. Le titre complet se lit comme suit: Relation abrégée de quelques missions des Pères de la Compagnie de Jésus dans la Nouvelle-France (Montréal, 1852). L'ouvrage italien s'intitule: Breve relatione d'alcune missioni de PP. della Compagnia di Gesù Nelle Nova Francia Del P. Francisco Giuseppe Bressani delle medisima Compagnia (Macerata, 1653). 
graver les cartes, lettrines et culs-de-lampe de ce livre. "Lovell a déjà fait faire ici un choix pour les gravures de Bressani, écrit le traducteur à Jacques Viger, le 13 juin 1851, et ce que je viens d'en voir est très encourageant. Il a un jeune graveur qui travaille bien ...". ${ }^{27}$

Le 6 juin précédent, le $\mathrm{P}$. Martin avait écrit à ce même Viger, en villégiature à Saint-Clément de Beauharnois : "J'ai envoyé mon Mss du P. Bressani à l'Orateur de la Chambre. Je ne sais pas si une assemblée à moitié française portera autant d'intérêt à un vieux monument d'une époque reculée que nos voisins les Yankees. J'attends avec impatience pour pouvoir procéder d'après cela à l'impression." 28 Il faut croire que les démarches n'ont subi aucun retard, puisque le 26 juin, il pouvait ajouter: "La complaisance de M. Faribault a pris fait et cause pour l'ouvrage du P. Bressani; ainsi que l'honorable Morin, ils l'ont présenté à plusieurs membres et je viens d'apprendre qu'il est très probable que je vais avoir la semaine prochaine une réponse favorable du Comité de la bibliothèque. Ils ont déjà fait une estimation ...".29

Nous savons par la correspondance du P. Martin que l'édition de cette Relation abrégée a coûté quatre mille francs et que le gouvernement en a versé pour sa part $1,800 .^{30} \mathrm{Si}$ le $\mathrm{P}$. Martin avait fait appel au gouver-

27. Une quinzaine de lettres du P. Félix Martin à Jacques Viger sont aujourd'hui conservées aux ASQ (archives du séminaire de Québec), boîte 21, liasse III. Viger, comme on le sait, a été un des plus actifs chercheurs de son temps. Très méthodique et d'une curiosité intellectuelle remarquable, il n'en conservait pas moins un grand respect pour les manuscrits. Il est entré en correspondance avec des centaines de chercheurs et il copiait les informations trouvées auprès d'eux dans les livres de sa Saberdache. M. Victor Morin qui a écrit "L'odyssée d'une société historıque" dans les Cabiers des Dix, 8 (1943) : 18-54, a consacré quelques pages aux manuscrits de Jacques Viger. Il a expliqué comment ces papiers sont passés successivement des mains des demoiselles Lennox, belles-filles de Viger, aux mains de l'abbé M.-A. Verreau, deuxième président de la Société historique de Montréal, puis à la soeur de ce dernier et enfin en Séminaire de Québec. L'abbé E.-G. Plante, de Québec, était membre de la Société historique de Montréal. Le 12 janvier 1864, il écrivait à Raphaël Bellemare, secrétaire de la Société historique, de lui faciliter "l'acquisition de la bibliothèque, des documents et des manuscrits de feu Jacques Viger". ASQ, Documents Plante, $\mathrm{n}^{\circ}$ 194. A propos de Verreau, on lira Armand Yon, L'abbé H.-A. Verreau, éducateur, polémiste, bistorien (Montréal, 1946) et Fernand Grenier, édit., Papiers Contrecoeur (Québec, 1952), 23.

28. ASQ, boîte 21, liasse III.

29. ASQ, boîte 21, liasse III.

30. Lettre du P. Martin au P. Coué, en France, le 24 décembre 1852. ACSM, cahier Litterae ad nostros. Cette lettre est citée par le P. Paul Desjardins, Collège Sainte-Marie de Montréal (2 vol., Montréal, 1940-42) 1: 222. 
nement, c'était parce que son expérience lui avait démontré que le public ne répondait pas à l'appel des écrivains. L'évêque de Québec, monseigneur Baillargeon, le lui rappelait bientôt le 15 février 1853: "Disons aussi, écrivait-il au P. Martin, que pour bien faire, il faudrait que ces volumes de la Relation du P. Bressani fussent reliés, et puis encore, que leur prix devrait être en raison inverse de leur mérite pour être conforme à la manière de raisonner de notre monde, tel qu'il est, c.-à-d. parcimonieux ... Tenez, dans notre siècle, on ne fait pas ce que l'on veut."' ${ }_{1}$

Cette expérience, Viger la partageait avec le P. Martin puisqu'il rapportait précisément cette phrase et la soulignait dans sa lettre à Faribault. Viger et Faribault étaient d'ardents amis de l'histoire qui s'intéressaient l'un et l'autre aux Relations des Jésuites du XVIIème siècle. Cette phrase avec ses soulignés prenait donc un sens tout particulier sous la plume du chercheur montréalais.

Ce n'était pourtant pas la première fois que Viger constatait l'apathie des Canadiens. Et chaque fois, il en était "confus et honteux" 32 comme le Corbeau de la fable, mais jamais il ne jura "qu'on ne l'y prendrait plus". Il avait bel et bien écrit le 29 juillet 1849 :

Voyez, comme on s'y prend autrement en Amérique qu'ici pour faire des collections historiques, et comment l'argent est prodigué : - 12,000 piastres mises à la disposition du Col. Brodhead. - 3,000, à celle de Mr Poore! C'est ça qui s'appelle faire les choses convenablement et sentir l'importance de l'oeuvre.

Vous voyez que $\mathrm{M}$. de $\mathrm{P}$ [uibusque] songe bien sérieusement à mes Archives du Canada comme il les appelle et vous voyez aussi comme on pourrait en peu de temps en faire quelque chose de considérable et de précieux, si l'on votait quelque somme et si l'on fesoit voyager à l'étranger, après avoir cherché et rassemblé ici. ${ }^{33}$

31. Ibid., $1: 221$. L'original de cette lettre est aux ACSM $n^{\circ}$ 3174-12.

32. Faribault était honteux d'avouer l'impossibilité de recueillir des abonnements à des revues françaises ou de trouver des souscripteurs pour le livre de Henry de Courcy: Les Servantes de Dieu en Canada. Dans une lettre du 24 mars 1853, il notait à Viger: "Ecoutez donc, il faut taire ces vérités à $M$. de Courcy - mais vous pouvez lui dire, ce qui est vrai aussi ..." ASQ, boîte 21, liasse III. On trouvera aussi dans le fonds Viger, ASQ, parmi les cahiers ou registres, aux numéros 72 et 73 , deux carnets intitulés : Souscriptions pour l'Histoire de l'Eglise aux Etats-Unis par Courcy et Souscriptions aux Servantes de Dieu en Canada.

33. APQ, Correspondance Viger-Faribault. 
Une fois passées ses justes colères, Viger recommençait de nouvelles expériences, parce que son amour désintéressé de la science lui faisait oublier ses propres échecs. Dans une lettre du 20 décembre 1852, nous pouvons le suivre dans une de ses réactions.

Un Mr John G. Shea, ci-devant novice ici sous le P. Martin vient de publier un superbe vol. à N. York, intitulé : Discovery and Exploration of the Mississipi Valley, with the original narratives of Marquette, Allouez Mss de Québec, Hennepin and Douay.

$A h$ ! ça, c'est du bon par exemple et même de l'excellent. Il y a quantité de Notes précieuses. Mais ce qui me choque pourtant, dans cette publication, c'est que notre Mss de Québec a été publié par un Yankee et à N. York, au lieu de l'avoir été par le P. Martin, ou un Canadien et ici, comme je préméditais de le faire. Mes Notes sont toutes prêtes, il y a longtemps. Mais nous sommes si gueux et si lents à tout faire, qu'après avoir fait mes petits reproches au cher Père de la livraison de son Mss à Mr Shea, j'ai fini par lui pardonner . . . et nous sommes toujours bons amis. ${ }^{34}$

Jacques Viger, qui n'était pas doux dans ses remontrances, a dû se rendre à l'évidence, car sa rancune même est tombée. Il continua d'être pour le P. Martin l'excellent ami que nous révèle toute sa correspondance avec Faribault. Ils continuèrent à préparer ensemble plusieurs travaux sur notre histoire et ils semblent par ailleurs avoir conservé à l'égard l'un de l'autre la plus entière indépendance de sentiments. On peut dire que c'est un cas unique dans la vie de Viger. ${ }^{35}$

$\mathrm{Au}$ sujet du même livre sur Marquette et Jolliet, l'abbé Ferland écrivait dans ses Notes sur les registres de Notre-Dame de Québec: "Voilà donc encore un des hommes les plus remarquables du Canada, tiré de l'oubli par un étranger : combien en est-il parmi les Canadiens instruits qui connaissent le sieur de Jolliet?... N'est-il pas surprenant qu'au

34. APQ, Correspondance Viger-Faribault. Les Notes de Jacques Viger concernant le manuscrit du P. Marquette sont au cahier F de la Saberdache rouge. Depuis quelques mois, il existe une copie en microfilm de la Saberdache à la salle Gagnon de la Bibliothèque municipale de Montréal. Plusieurs cahiers de la Saberdache ont été recopiés par Viger lui-même qui en a fait don à son ami et collaborateur, le P. Félix Martin. Ils sont aujourd'hui conservés aux ACSM.

35. Guy Frégault, "La recherche historique au temps de Garneau (La correspondance Viger-Faribault)", Centenaire de l'Histoire du Canada de François-Xavier Garneau, 371-90. Aussi Thomas Charland, "A qui devons-nous la réédition des Relations des Jésuites ?" RHAF, 3 (1949) : 215-9. 
défaut des enfants de notre sol, des écrivains n'appartenant pas à notre pays soient obligés de nous rappeler ces noms ?"36

Avons-nous remarqué, en quelques mois ou quelques années, cette espèce d'obsession des Yankees ? Le 6 juin 1851, le P. Martin s'inquiète de savoir "si une assemblée à moitié française portera autant d'intérêt à un vieux monument d'une époque reculée que nos voisins les Yankees". Quelques mois plus tard, c'est Viger qui se choque de voir que "notre Mss de Québec a été publié par un Yankee et à N. York". Il doit pourtant se "déchoquer" pour de bonnes raisons et d'ailleurs il a lui-même déjà louché vers 1" Amérique" et son savoir-faire pour la chose historique. Et dans ses Notes sur les registres, l'abbé Ferland s'étonne à son tour : "Voilà donc encore un des hommes les plus remarquables du Canada, tiré de l'oubli par un étranger." Ferland, comme Viger, ne semble pas à sa première expérience. S'il est surpris, il doit conclure “qu'au défaut des enfants de notre sol, des écrivains n'appartenant pas à notre pays soient obligés de nous rappeler ces noms ?"

Quelle sorte d'intérêt montraient donc les Yankees pour l'histoire du Canada ? En 1837, Georges Bancroft, en 1845, Jared Sparks, en 1846 et 1868, Francis Parkman venaient consulter et copier les manuscrits de la Société littéraire et historique de Québec. Le Dr E.B. O'Callaghan, après avoir quitté le Canada pour habiter New-York, venait de terminer l'édition des Documents relating to the History of the Colony (1604-1799). C'était une considérable compilation de pièces d'histoire concernant ì la fois les colonies américaines et le Canada. Durant les années 1854 et 1855, James Lenox éditait à Albany le Narré du voyage du P. Druillettes, le Récit des voyages et découvertes du R. P. Jacques Marquette en l'année 1673 et la Relation de ce qui s'est passé . . en 1676 et 1677 . Vers la même époque, Shea et O'Callaghan commençaient la réimpression du vieux manuscrit sur une presse Cramoisy qu'ils avaient fait préparer pour reproduire ces anciens documents selon les caractères typographiques de l'imprimeur des Relations de la Nouvelle-France. C'était un tirage luxueux fait à 25 ou 100 exemplaires. ${ }^{37}$

Dans tous les états de l'Union américaine, on assistait à une effloraison toujours plus grande de sociétés historiques. Nous connaissons

36. Notes sur les registres ..., 35

37. Thwaites, éd., Jesuit Relations (73 vol., Cleveland, 1896-1901), 5: 280. 
aussi le cas d'un riche particulier, marchand new-yorkais, James Lenox, devenu subitement multimillionnaire par la vente de terrains. Il occupait ses loisirs et sa fortune à acheter et à collectionner une remarquable série de livres sur l'histoire du Canada et de l'Amérique. Henry Stevens qui fut son acheteur de livres à Londres pendant trente-cinq ans, nous assure que durant la seule année 1854-1855, James Lenox a dépensé cinquante mille dollars en achat de livres. En 1886, ajoute-t-il, le même travail coûterait 250,000 dollars. ${ }^{38}$

Et cet exemple de Lenox n'est pas unique. On peut encore citer $\mathbf{M}$. Gallatin de New-York, l'honorable H.C. Murphy de Brooklyn, O.H. Marshall de Buffalo, John Carter Brown de Providence, J.C. Fisher de Philadelphie, B.U. Campbell d'Ellicot's Mills, Maryland. ${ }^{39}$ Tous ces noms sont connus des collectionneurs américains puisqu'ils se trouvent attachés aux sections de bibliothèques publiques héritières de leurs chers livres. Même les catalogues préparés par ces vieux bibliophiles sont considérés de nos jours comme de précieux répertoires bibliographiques.

A la même époque, la Société historique de New-York permettait l'accès de ses documents à Félix Glackmeyer, de la Société littéraire et historique de Québec, qui en 1845 en rapportait dix-sept gros volumes in-folio de copies. ${ }^{40}$ En France, l'accueil était un peu différent. On lit dans Justin Winsor: "Henry Harisse rapporte que le gouvernement français a refusé l'accès à ses archives à un agent de la Société historique de Québec en 1835 , et qu'un semblable refus a été fait en 1838; mais qu'en 1842, le général Cass, ministre des E.-U. a réussi, en faveur de l'Etat du Michigan, à obtenir quarante cartons pour la publication." Justin Winsor rapporte encore un autre témoignage: "Louis-P. Turcotte dit que les premiers essais tentés pour obtenir des copies des archives européennes en 1835 n'ont pas réussi dans le temps et que de meilleurs résultats ont été obtenus par l'Etat de New York (1841-1844). En 1845, un agent canadien, M. Papineau, s'est procuré d'autres copies en France et en 1851-52, M. Faribault a pu ajouter vingt-quatre volumes de copies à sa collection." 41

38. Henry Stevens, Recollections of Mr James Lenox of New York and the foundation of bis library (London, 1896), 96.

39. Les répertoires bibliographiques de l'époque nous le montrent clairement. A citer, par exemple, Justin Winsor, Narrative and Critical History of America.

40. Philéas Gagnon, Essai de bibliographie canadienne, I : 354, $\mathrm{n}^{\circ} 2571$.

41. Justin Winsor, Narrative and Critical ..., 4:366. Nous avons cité précédemment une lettre de Jacques Viger faisant allusion aux mêmes faits et aux mêmes personnages. 
En 1856, l'abbé Ferland se rend en France à son tour pour recueillir des documents de notre histoire. Dans son Journal de voyage, il note à la date du 27 juin 1856, qu'il a pénétré mais avec difficulté aux Archives de la Marine et à celles de la Guerre. Là, il a été obligé pour faire ses copies de s'en tenir à l'usage du crayon. ${ }^{42}$

Arrêtons ici notre enquête. Nous y avons recueilli assez de renseignements sur les circonstances qui ont entouré cette réédition des Relations des Jéswites. Ces circonstances ressemblent évidemment à bien d'autres, mais elles nous éclairent sur une époque. Venons-en maintenant à l'étude de la motion parlementaire qui a autorisé la participation financière du pouvoir public.

Le Journal de Québec écrivait le 11 décembre 1858 que "des amis de l'entreprise s'adressèrent à l'honorable Morin, alors ministre, qui promit au nom du gouvernement une part de coopération". Morin était sans doute le premier homme à rencontrer à cause du poste qu'il occupait, mais aussi à cause des dispositions qu'il avait plus d'une fois manifestées. N'avait-il pas écrit en 1823 dans le programme de la Minerve: "L'histoire de notre pays sera aussi un des objets principaux de nos recherches. Nous prions ceux qui connaissent d'anciennes productions canadiennes, de vouloir bien nous les communiquer, afin de les soumettre à la critique avant que les monuments qui peuvent servir à leur examen disparaissent entièrement." 43 En 1831, il avait lui-même occasion d'appliquer ce programme. Il obtenait de la Chambre une somme de $\$ 500$ pour la publication de l'Histoire du Canada du Dr Labrie, décédé peu auparavant. En 1852, nous l'avons vu intervenir à la Chambre en faveur de la Relation abrégée $d u \quad P$. Bressani. Et maintenant comme chef parlementaire, n'était-il pas l'homme le plus disposé à appuyer le projet de réédition des Relations?

Au premier rang des "amis de l'entreprise", nous voyons très bien G.-B. Faribault, d'abord à cause de l'amitié qui l'unissait à Morin, amitié qui fut plus d'une fois utilisée à l'avantage d'autres projets de même nature. C'est auprès de ces deux hommes, unis dans l'opinion publique,

42. Archives de l'archevêché de Québec (AAQ), Armoire E, rayon 11, papiers Ferland, $\mathbf{n}^{\circ} 12$.

43. Ce texte est rapporté par A. Béchard, L'bonorable A.-N. Morin, 54. 
que l'abbé Laverdière commencera en effet sa campagne pour rééditer les Oeuvres de Champlain,44 un peu plus tard. Une autre raison nous fait désigner Faribault et c'est le texte même de la motion. On y propose de faire réimprimer "les Documents qui ont été obtenus et extraits des Archives publiques à Paris et à Londres". Est-ce une allusion au voyage que Faribault faisait en Europe en 1852 ? Il semble, puisque la motion ajoute immédiatement que ces mêmes documents "sont maintenant conservés en manuscrits dans la Bibliothèque du Parlement ou dans la Bibliothèque de la Société littéraire et historique de Québec". Nous ne sommes donc pas en face d'une simple formule, mais d'une allusion directe à un seul et même homme qui occupe les deux postes mentionnés.

Après le nom de Faribault, nous placerions volontiers ceux des abbés Laverdière et Ferland. Mêlés l'un et l'autre très activement à la réalisation de cette édition, ils ont assurément participé aux préparatifs lointains, c'est-à-dire à ces approches nécessaires à l'adoption d'une motion en Chambre. Nous sommes presque confirmés sur le rôle de Laverdière par une lettre que l'imprimeur Auguste Côté lui adressait le 22 décembre 1858: "Je vous envoie mon mémoire, écrivait-il, pour le lire et vous prie de donner aujourd'hui, si faire se peut, votre lettre d'appui devant le gouvernement car j'ai besoin de ne pas manquer mon coup." 45 En effet, la circonstance est importante, même si elle est de 1858. Voici les faits. Pour avoir ajouté quelques documents supplémentaires à la série des Relations, l'imprimeur se voit menacé par le sous-secrétaire provincial, Etienne Parent, de n'être pas remboursé pour des dépenses prétendues imprévues et non autorisées. Que fait immédiatement l'imprimeur ? Il se tourne instinctivement vers Laverdière, l'homme important et puissant dans cette affaire. Et Laverdière entre directement en intervention auprès d'Etienne Parent. ${ }^{46}$

Quant à l'abbé Ferland, il n'était pas un inconnu lui non plus. Déjà il avait commencé ses travaux historiques et en avait publié quelques-uns. Quand il se rendra en Europe en 1856, il ira jusqu'à Rome pour trouver des volumes des Relations. A.-N. Morin ne se tournera-t-il pas vers lui, par une alliance d'idées ou un simple désir d'obtenir un échange de servi-

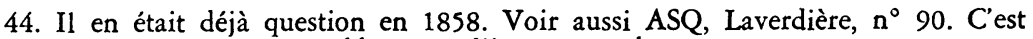
une lettre du P. F. Martin à l'abbé Laverdière, 29 octobre 1858.

45. ASQ, Laverdière, $\mathrm{n}^{\circ} 52$.

46. ASQ, Laverdière, nos 95 et 96. 
ces, quand il lui écrira le 14 décembre 1859 ? ${ }^{47}$ Il s'agit encore d'une édition des Relations, mais cette fois en anglais et chez John Lovell de Montréal. Morin recommande à Ferland le nom du Dr William Wilson, traducteur de la Chambre, pour accomplir la version de ces textes historiques. $^{48}$ En 1859, Morin est juge et professeur de droit à l'Université Laval, mais en septembre 1854, ne s'était-il pas vu décerner un doctorat bonoris causa en droit à l'occasion de l'inauguration de la même université ? ${ }^{49}$ C'était deux semaines avant la motion du 2 octobre.

Et ce n'est pas tout. Les deux prêtres étaient encore bien placés aux yeux du gouvernement, de la Chambre et de la bibliothèque parlementaire, pour recommander, presser la réédition des Relations en proposant l'adoption d'une motion qui serait votée en Chambre le 2 octobre 1854. Tous les livres de la bibliothèque qui avaient échappé à l'incendie de février de la même année, logeaient encore dans les "Bâtiments du Séminaire". C'était même grâce à la collaboration du personnel entier du Séminaire, prêtres et élèves, que les 17,500 volumes n'avaient pas brûlé cette nuit-là. Le parlement se le rappelait puisque, le 18 décembre suivant, il empruntait la plume de l'orateur de la Chambre pour transmettre ses remerciements à l'institution. Et quatre jours plus tôt, il avait voté une motion dans le même sens. ${ }^{50}$

Quoi qu'il en soit de nos conjectures présentes, la Chambre adopta une motion le 2 octobre 1854. Le texte de la motion ne serait pas facile à retracer aujourd'hui dans le Journal de l'Assemblée législative s'il n'avait pas été publié et identifié comme tel par les journaux de l'époque. En cela, nous pouvons dire que la dispute de décembre 1858 nous aura rendu service. La dite motion ne mentionnait pas de façon explicite les Relations des Jésuites. Elle était même si vague dans sa teneur que l'application pourrait s'en faire à n'importe quel autre document imprimé ou manuscrit; ce n'était peut-être pas sans raison. Qu'on en juge immédiatement par soi-même:

47. Cette lettre fait partie du fonds Gagnon, à la bibliothèque municipale de Montréal. Philéas Gagnon l'a signalée dans son Essai de bibliographie canadienne, I : 414 .

48. ACSM, prospectus de cette édition en projet, ainsi qu'une lettre de Mlle Wilson qui offrait au collège la traduction manuscrite préparée par son père. On sait que le projet d'une édition anglaise n'a pas eu de suite.

49. A. Béchard, L'Honorable A.-N. Morin, 178.

50. Archives de la cure Notre-Dame de Québec, carton 25, nos 20 et 20A. Le document rapporte que tout le personnel du Séminaire a participé au sauvetage. 
Sur motion de l'Honorable M. Chauveau, secondé par l'Hon. M. Chabot,

Résolu: Qu'il soit présenté une humble adresse à son Excellence le Gouverneur-Général, priant son Excellence de faire imprimer, en sus des Documents mentionnés dans une Adresse à cette Chambre, les Documents qui ont été obtenus et extraits des Archives publiques, à Paris et à Londres, et qui sont maintenant en manuscrit dans la Bibliothèque du Parlement, ou dans la Bibliothèque de la Société littéraire et historique de Québec, ou qu'on pourra se procurer par la suite, et qui paraîtront d'un intérêt suffisant au point de vue légal et historique; et aussi, de faire ré-imprimer ceux des ouvrages publiés dans les premiers temps de la Colonie, et qui peuvent être d'une grande valeur et sont devenus très rares, les dits ouvrages et Documents devant être imprimés dans la forme et avec les notes et cartes qui seront jugés convenables, et assurer son Excellence que cette Chambre s'empressera de subvenir aux dépenses nécessaires pour l'accomplissement des objets susdits.

Ordonné, que la dite Adresse soit présentée à son Excellence le Gouverneur-Général, etc., etc. ${ }^{51}$

Servons-nous tout de même des données de ce texte pour éclaircir notre problème. Chauveau et Chabot étaient des politiciens de carrière qui travaillaient depuis longtemps aux côtés de Morin. Choisis une première fois en 1851 comme responsables de deux des cinq portefeuilles dans le gouvernement Hincks-Morin, ils avaient été désignés de nouveau en septembre 1854 comme membres du cabinet McNab-Morin. ${ }^{52}$

La campagne électorale qui avait porté le parti au pouvoir s'était déroulée au cours des mois de juillet et d'août 1854. Elle avait été, paraît-il, d'une violence vraiment remarquable. "De tous les députés hostiles au gouvernement Hincks-Morin, celui qui manifesta son dépit le plus ouvertement fut M. Cauchon",53 dans le Journal de Québec où il était rédacteur. ${ }^{54}$ Voilà qui permet d'expliquer la rivalité qui opposa d'une part le Journal de Québec, aux ateliers duquel furent imprimées les Relations des Jésuites, et d'autre part, le Courrier du Canada, qu'on disait à la

51. Journal de l'Assemblée législative, 1854-55, 5.

52. A. Béchard, L'Honorable A.-N. Morin, 170; Thomas Chapais, Cours d'bistoire d* Canada ( 8 vol., Québec, 1919-34), 7 : chap. 1, 2 et 3.

53. A. Béchard, L'Honorable A.-N. Morin, 166, note; Thomas Chapais, Cours d'bistoire du Canada, $7: 55$.

54. Pierre-Georges Roy, A Propos de Crémazie (Québec, 1945), 81. 
solde de P.-J.-O. Chauveau, parrain de la motion du 2 octobre 1854. Mais la querelle était déjà vieille et les Relations ne furent que l'occasion de la ranimer.

Pour arracher à Chauveau la part du lion qu'il réclamait par l'intermédiaire du Courrier du Canada, le journal rival se donnait la peine de préciser certains faits. "C'est à la suite de la promesse de M. Morin que M. Chauveau proposa la résolution". Bien plus, ajoutait le rédacteur du Journal de Québec, "c'est parce que M. Morin avait promis que ses successeurs, et notamment $M$. Cartier, alors secrétaire provincial, donnaient l'encouragement libéral qui a si puissamment aidé au succès de la publication canadienne jusqu'ici la plus considérable".

Rappelons-nous les événements survenus de 1854 à 1858 . Les sessions de ces années étaient toujours longues et fort occupées par la discussion des problèmes les plus importants pour l'avenir du pays. "Cette session si importante, note Thomas Chapais, ${ }^{55}$ commencée le 5 septembre 1854, ajournée le 18 décembre, reprise le 23 février 1855, se termina le 30 mai. Elle avait vu tomber un ministère et naître un gouvernement nouveau. Elle avait assisté à une évolution d'alliances et à un remaniement des partis. Elle avait inscrit dans les statuts du pays des acles législatifs de la plus haute portée économique et sociale..."

Le départ de lord Elgin, gouverneur-général, avait fait suspendre la session le 18 décembre. La démission de l'Honorable Morin, pour cause de santé, avait amené la démission des quatre ministres ou comtnissaires du Bas-Canada. A la demande de sir Edmund Head, nouveau gouverneur, l'honorable E.-P. Taché reprenait son portefeuille de receveur général et assumait la direction du gouvernement du Bas-Canada. L'honorable Drummond était réinstallé procureur-général, mais les travaux publics passaient des mains de l'honorable Chabot à celles de François Lemieux, et le secrétariat provincial était confié à Georges-Etienne Cartier. Chauveau, ancien secrétaire provincial, devenait surintendant de l'instruction publique au Bas-Canada à la place du Dr J.-B. Meilleur. Morin, universellement regretté comme homme politique, était nommé juge à la cour supérieure et Chabot recevait la charge de la commission de la tenure seigneuriale.

55. Thomas Chapais, Cours d'bistoire du Canada, $7: 113$. 
La session suivante s'ouvrit à Toronto, le 15 février 1856. Trois mois plus tard, Georges-Etienne Cartier cessait à son tour d'être secrétaire provincial, avec la chute du ministère $\mathrm{McNab}$. Voilà pour les mutations parlementaires.

Ordinairement les projets d'aide aux éditions de livres, recevaient une première approbation en Chambre, puis étaient soumis à l'étude du comité de la bibliothèque. Si la demande était jugée convenable, le gouvernement promettait alors d'acheter un certain nombre d'exemplaires pour en faire lui-même la distribution gratuite. Lisons le Journal de Québec du 11 décembre 1858 :

Le gouvernement s'est engagé à prendre mille exemplaires des Relations, si M. Côté les publiait, et ces mille exemplaires lui ont été délivrés. En cela le gouvernement a fait ce qu'il fait pour toutes les publications importantes. C'est ainsi qu'on a pris un certain nombre d'exemplaires de l'histoire du Canada de $M$. Christie et des deux premières éditions de l'histoire de $M$. Garneau, et que l'on s'est engagé à prendre un certain nombre aussi de la troisième édition du même et important ouvrage... Les Relations des Jésuites étaient plus considérables, le gouvernement donnait davantage, donnait assez pour rendre la publication possible...

Le P. Léon Pouliot affirme que le gouvernement "y affectait la somme de 1,400 livres". ${ }^{56}$ Voilà qui nous donne le montant global de la facture et approximativement le coût de revient d'un exemplaire. Le secrétaire provincial qui les distribuait ensuite faisait un cadeau d'environ une livre et huit shellings la série. Si on évalue la livre à $\$ 4.00$, la contribution gouvernementale atteignait les $\$ 5,600$. C'est par quatre ou cinq qu'il faudrait aujourd'hui multiplier ce montant pour avoir une juste idée du coût actuel.

Mais il convient d'ajouter les trois cents exemplaires réservés à l'éditeur ou imprimeur pour se dédommager de ses dépenses. Augustin Côté vendait les Relations des Jésuites deux livres la série, soit $\$ 8.00$, affirme l'abbé Bois. ${ }^{57}$ De nos jours, ces trois volumes se vendent entre $\$ 30$ et $\$ 40$. Déduisons pour l'imprimeur une cinquantaine de séries distri-

56. Léon Pouliot, Etude sur les Relations, 32.

57. Archives du collège de Nicolet, fonds Bois, correspondance, cahier 17 bis : lettre du 22 septembre 1858, "au prix bien modique de $£ 2$ ". 
buées à ses divers collaborateurs, et il lui reste près d'un millier de dollars. Il y a peut-être lieu d'ajouter quelques contributions personnelles, plutôt rares, et l'édition aura coûté en chiffres ronds $\$ 6,000$ ou $\$ 7,000$.

Et nous avons terminé l'étude préliminaire des circonstances. Cette réédition avait son importance, quoique toute relative. Dans la vie courante et dans la politique, elle ne devait certainement pas tenir le centre des préoccupations, surtout à un moment où l'on songeait par exemple à fixer le siège du gouvernement canadien, où l'on discutait l'ádoption d'un système monétaire uniforme, l'établissement des chemins de fer et divers autres problèmes importants pour l'avenir du pays.

Tout de même nous avons pu y voir une manifestation de la vie culturelle, née de l'initiative privée et secondée par la finance publique. "Les ouvrages d'utilité pratique et publique ont pu être édités à la faveur d'allocation du gouvernement, écrivait Edmond Lareau en 1877, mais rarement avant ces vingt dernières années, avons-nous constaté l'apparition d'un livre lancé dans le public par l'initiative individuelle." ${ }^{8} \mathrm{Si}$ l'auteur entend par "initiative individuelle" les risques financiers, son observation semble assez vraie. Car l'exemple des Relations des Jésuites n'est pas unique, nous l'avons vu, mais il vient se placer tout naturellement dans le mouvement historiographique encore naissant au Canada à cette époque.

Collège Sainte-Marie, Montréal.

Georges-Émile GiguÈre, S.J.

N.B. Cet article est le chapitre préliminaire d'une thèse sur l'édition canadienne des Relations des Jésuites.

58. Mélanges bistoriques et littéraires (Montréal, 1877), 2. 